\title{
Stratégies de complémentation des ovins de race Djallonké en phase de croissance
}

\author{
TYo
}

IDESSA, Département des Ressources Animales, BP 633 Bouaké, Côte d'ivoire

Dans les zones humides et subhumides de l'Afrique occidentale, l'élevage ovin traditionnel utilise essentiellement des animaux de race "Djallonké", du fait de leur trypanotolérance. Ces animaux de petit format (poids adulte $=25$ à $35 \mathrm{~kg}$ ) ont une vitesse de croissance faible avec un GMQ rarement supérieur à $90 \mathrm{~g} / \mathrm{j}$ après le sevrage (Berger et Ginisty, 1980, Rev Elev Med Vet Pays Trop, 33, 71).

Des essais ont été menés pour déterminer l'efficience alimentaire du tourteau de coton ou de feuilles d'arbres fourragers, comme complément protéique chez des agneaux sevrés âgés de 3 à 4 mois et pesant 10 à $14 \mathrm{~kg}$. Dans une première étude, les animaux ont pâturé sur une culture fourragère fertilisée (Panicum maximum, $14 \%$ MAT), et ont reçu comme supplément du tourteau de coton ou des feuilles de Pterocarpus erinaceus ou Bridelia ferruginea. Dans une seconde série d'études, l'aliment de base était constitué par un fourrage grossier: paille de Brachiaria ruziziensis ou de Panicum maximum (5 à $6 \%$ MAT). Les lots expérimentaux ont reçu, comme supplément, du tourteau de coton ou des feuilles de fourrages ligneux (Albizzia zygia, Ficus exasperata ou Morinda lucida). Les principaux résultats sont résumés dans le tableau.

Une complémentation à base de tourteau de coton permet aux agneaux de race Djallonké en post-sevrage d'extérioriser leur potentiel de croissance. Les fourrages ligneux peuvent être utilisés pour complémenter les aliments grossiers ; ils permettent une croissance modérée et réduisent les taux de mortalité.
Régime de base

Panicum vert

Panicum vert

Panicum vert

Panicum vert

Paille Brachiaria + $100 \mathrm{~g}$ mélasse

Paille Brachiaria + $100 \mathrm{~g}$ mélasse

Paille Brachiaria $+100 \mathrm{~g}$ mélasse

Paille Brachiaria $+100 \mathrm{~g}$ mélasse

Paille Panicum $+100 \mathrm{~g}$ mélasse

Paille Panicum $+100 \mathrm{~g}$ mélasse

Paille Panicum + $100 \mathrm{~g}$ mélasse

Paille Panicum $+50 \mathrm{~g}$ t. coton

Paille Panicum $+50 \mathrm{~g}$ t. coton

Paille Panicum $+50 \mathrm{~g}$ t. coton supplément

tourteau coton
Pterocarpus
Bridelia

tourteau coton

MS suppl

g/

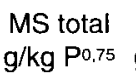

MAT $g / k g P^{0,75}$

tourteau coton

Albizia

tourteau coton

Ficus

36

52

$\begin{array}{ll}- & \\ - & \\ - & \\ - & \end{array}$

/kg MS

$\begin{array}{cc}\text { GMQ } & \text { Mor } \\ 9 / \mathrm{j} & \% \\ 47^{\mathrm{a}} & 0 \\ 88^{\mathrm{b}} & 10 \\ 43^{\mathrm{a}} & 10 \\ 51^{\mathrm{a}} & 0 \\ & \\ 6^{\mathrm{a}} & 18 \\ 93^{\mathrm{c}} & 0 \\ 113^{\mathrm{d}} & 0 \\ 31^{\mathrm{b}} & 0\end{array}$

Morinda

$-$

Morinda

\begin{tabular}{cccc}
58 & 53 & $6^{\mathrm{a}}$ & 18 \\
81 & 159 & $93^{\mathrm{c}}$ & 0 \\
93 & 241 & $113^{\mathrm{d}}$ & 0 \\
91 & 124 & $31^{\mathrm{b}}$ & 0 \\
& & & \\
63 & 50 & $-49^{\mathrm{a}}$ & 63 \\
89 & 211 & $74^{\mathrm{c}}$ & 0 \\
89 & 119 & $38^{\mathrm{b}}$ & 12 \\
& & & \\
63 & 97 & $7^{\mathrm{a}}$ & 50 \\
81 & 134 & $26^{\mathrm{b}}$ & 17 \\
87 & 166 & $40^{\mathrm{b}}$ & 0 \\
\hline
\end{tabular}

